\title{
Crime Analysis and Intelligence System Model Design using Big Data
}

\author{
Abubakar Aliyu Machina \\ Research Scholar, School of Computer Science \& \\ Information Technology, Changchun University of \\ Science and Technology \\ Changchun, Jilin Province, China
}

\author{
Li Songjiang \\ Dr, Senior Lecturer, School of Computer Science \& \\ Information Technology, \\ Changchun University of Science and Technology \\ Changchun, Jilin Province, China
}

\begin{abstract}
The advent of new trend in information and communication technology specifically data science, machine learning and artificial intelligence unleashed various opportunities and offers solution to distinct level of problems in various domains. Globally, many countries have adopted the use of data driven technologies and crime analysis to predict and handle crime patterns and logics. However, most countries under-use this technology by using conventional or traditional techniques in crime analysis. A large amount of data is rapidly generated by various agencies of the government and independent organizations especially in Nigeria; agencies share common objectives or mandates. Other contributing factor is enforcement personnel ratio to the total population density. Thus, technology is required to complement the lack of adequate personnel. As a result, this research is aimed at designing a crime analysis and intelligence model using big data. Document Analysis, Questionnaire, and Interview is used to collect data from various law enforcement agents. Microsoft excel is used to generate accurate result and visualized the result in form of a pie chart, while UML models are used to depict logical and physical schema of the proposed model. The results analysis supports the hypothesis of the research by revealing the manual and traditional techniques of policing and crime analysis. Most of the agencies operate and keep track of records manually, while technology is applied to track cellphones. Moreover, a high majority of the respondents lack basic computer literacy and modern crime analysis techniques and big data. Hence, most of the respondent wish to adopt the use of big data analytics.
\end{abstract}

\section{Keywords}

Crime Analysis, Intelligence Systems, Model Design, Big Data

\section{INTRODUCTION}

Data Science as a multi-disciplinary field that employ the use of scientific methods, processes, algorithm and systems to extract knowledge and insights from structured and unstructured data integrating various skill set such as mathematics, machine learning, artificial intelligence, statistics, big data, database and optimization coupled with deep understanding of problem formulation to engineer effective solutions [1]. Applications of different data analytics technologies to security and criminal investigations contributed to the criminal analytics [2].

The advent of new trends in technology has turned data into new gold of the future. The increasing number of new IoT devices and other services has made the need of data dire in recent years. Data is being collected from numerous sources and in various format. revolution in information and communication systems, crime patterns have also taken a smart and data driven approach. Most crime can be associated to one or more forms of data with organized teams and roles. Many researches add value to this field by drafting some important contributions Tyagi et al, offers an approach to crime data analysis using a systematic review [3] and Barbutu et al discuss big data and policing [4], while Srinivas et al., proposed a crime analysis prediction system using big data [5], and Pramanik et al, deliberated on big data analytics for security and criminal investigations [2]. Sanjana et al, used big data approach for crime classification and visualization using crime data set [6], Feng et al, used big data analytics and mining for crime data analysis, visualization and prediction. Other relative contributions includes crime data analysis using pig with Hadoop [7], and Big data prediction on crime detection [8]. For further understanding [9] and [10] discussed Security issues associated with big data and big data and its technical challenges respectively.

Despite the valuable contributions of the previous work, application of big data for criminal analysis is possible if there exist an electronic format data set which can be used for crime data analysis; Hence, in Nigerian context most of the security and law enforcement agencies managed data and or crime information manually. Therefore, the main purpose of this work is to analyze and propose a novel solution that can be adopted and used in the near future and proposed a model that offers unified process for analyzing, reporting, tracking, accessing and sharing intelligent information across relevant authorities.

\subsection{Background of the Problem}

In today's world especially in the African Nations many sectors where facing serious challenges most of which are IT related. Realizing these problems earlier and quickly coping with current trend in Technology will help in the long run. Nigeria has many law enforcement and Intelligent agencies some of which share similar roles and responsibilities in safe guarding life and properties of the Nation and its citizens. Though, has the most alarming crime rate in the world [11] Nigeria is becoming unsafe in recent years due to the increasing rate of crime and crime related activities [12]. Therefore, crime prevention and analysis are important especially in this time and age.

In addition to the existing criminal activities new criminal activities are manifesting rapidly in various forms. Boko Haram killings, Fulani herdsmen attack or ambush, kidnapping, smuggling, drugs and arms trafficking via the porous and unguard boarders and sea port are commonly on the rise. other web related crimes such as online fraud, defamation of characters, fake news and all forms of web related crimes are also growing rapidly. 
Hence, the above-mentioned problems can be attributed to many factors to mentioned but a few related to information and communication technology are;

1. Lack of adequate manpower.

2. Underutilization of technology such as CCTV Camera, Automatic Plate Number Recognition etc.

3. Manual crime related record management.

4. Traditional or conventional patrol system.

5. Lack of trending crime analytics tools such as big data, artificial intelligence and machine learning.

\subsection{The Existing System}

Despite the high number of law enforcement agencies in the country, yet the ratio of law enforcement agent to citizens are highly outnumbered with many flaws at various level of the system. Recently, Nigeria has been contemplating on the emergence of State police due to the lack of enough personnel to carter for the security of the Nation. Alternatively, computers can do background work and make decisions intelligently and automatically [1].

According to Sheriff Deputies there are 3 major sources of
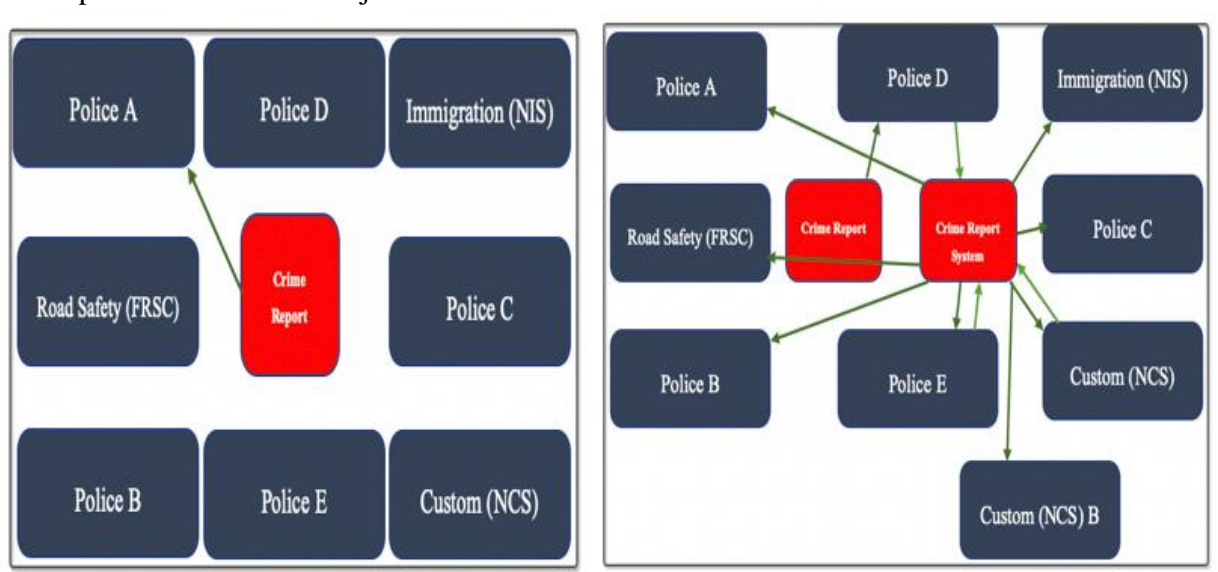

Figure 1 Comparison of Shared and Unshared Data systems

The figure 1 above depict an image where crime is reported to police department $\mathrm{A}$ and the information is only available to Police A and only Police A. Comparatively the second scenario illustrates an instance of a shared data or information where crime is reported to Police D, E and Custom Service A the information is shared to all relative enforcement agencies as options for restrictions will be available to the party sharing the report or information in the system. Thus, when taking relevant action, the responsible department has to be referred (i.e. Police D, E and Custom Service A).

\section{LITERATURE REVIEW}

There have been various ideas on the term big data. However, all the related definitions can be narrowed to voluminous and complex collections of data generated from different sources such as sensors, surveillance cameras, transaction machines or histories, databanks, national databases etc. which are difficult to process using conventional or traditional analytic tools [15], [16]. The definition can be extended using five important characteristics; Volume denotes huge voluminous data in order of terabytes and petabytes or even a higher degree, Velocity signifies the high velocity in which data is generated, Variety refers to the huge variety or types of data which can be structured, semi-structured or unstructured, Value denotes the intrinsic value of the data, Veracity refers to the gathering crime information in Nigeria, namely official statistics, victimization survey, and self-reported crimes survey [13]. Official statistics are not always accurate as they involve human interventions, while victimization survey is not the complete incidents as individual are afraid to reveal their identity as to the consequences. Therefore, this information suggests that the Most of Most of the agencies do not utilized the use of basic technologies such as Relational Database Management System (RDBMS), National Emergency or Crime Reporting systems such as the Rapid Response System commonly used in developed nations, smart camera, Automatic Plate Number Recognition Systems, National Crime Record Repository are all not in place.

Moreover, the NPF as the main body saddled with the responsibility of tackling crime lacks good and efficient coordination with other federal law enforcement agencies like NSCDC, EFCC, NIA, DSS etc. [14].

The most important problem of the existing systems is lack of intelligence or information sharing among various law enforcement agencies. Criminal explore this weakness by committing crime in different part of the nation through switching states and locations. An instance of this can be described in the figure below:

uncertainties in big data such as missing, duplicate and or incomplete entries [7].

One of the most powerful tools with great ability if applied correctly to prevent, reduce, and control crime and disorder is Crime Analysis [17]. According to the International Association of Crime Analysts (IACA) Crime analysis is the process in which a set of quantitative and qualitative techniques are used to analyze data valuable to police agencies and their communities, It also includes the analysis of crime and criminals, crime victims, disorder, quality of life issues, traffic issues, and internal police operations, and its results support criminal investigation and prosecution, patrol activities, crime prevention and reduction strategies, problem solving, and the evaluation of police efforts [18]. Processing can be done through writing codes that reads and executes procedures and return the queried information or files [19].

Therefore, crime analysis and big data refer to the tools and techniques used for transforming raw crime data into meaningful and crucial information which helps in forming a decision support system for relevant authorities or law enforcement agencies to take steps towards checking and investigating crimes prior to its occurrence. Hence, certain trends are discovered [20] Predictions and Analysis of crime are becoming more accurate and interesting with the advent of 
Big Data Tools [21]. Manning deduced that analytic ability of crime analysis and mapping could re-orient policing to anticipatory and preventive actions instead of call-for-service [17].

Though, analysis of large scale data set has been a challenging task [22], [23] many studies have discovered and implemented variety of techniques to investigate and discover new trend in crime data [15]. Since, enforcement agencies, intelligence agencies and the police maintain and manage their independent crime databases. The specifications and requirement of agencies differs whereas some share the same specifications but different requirements, as such data generated by both intelligence and enforcement agencies can be used as single entity. Though, Crime Analysis is of different types and levels as shown in figure 2 below;

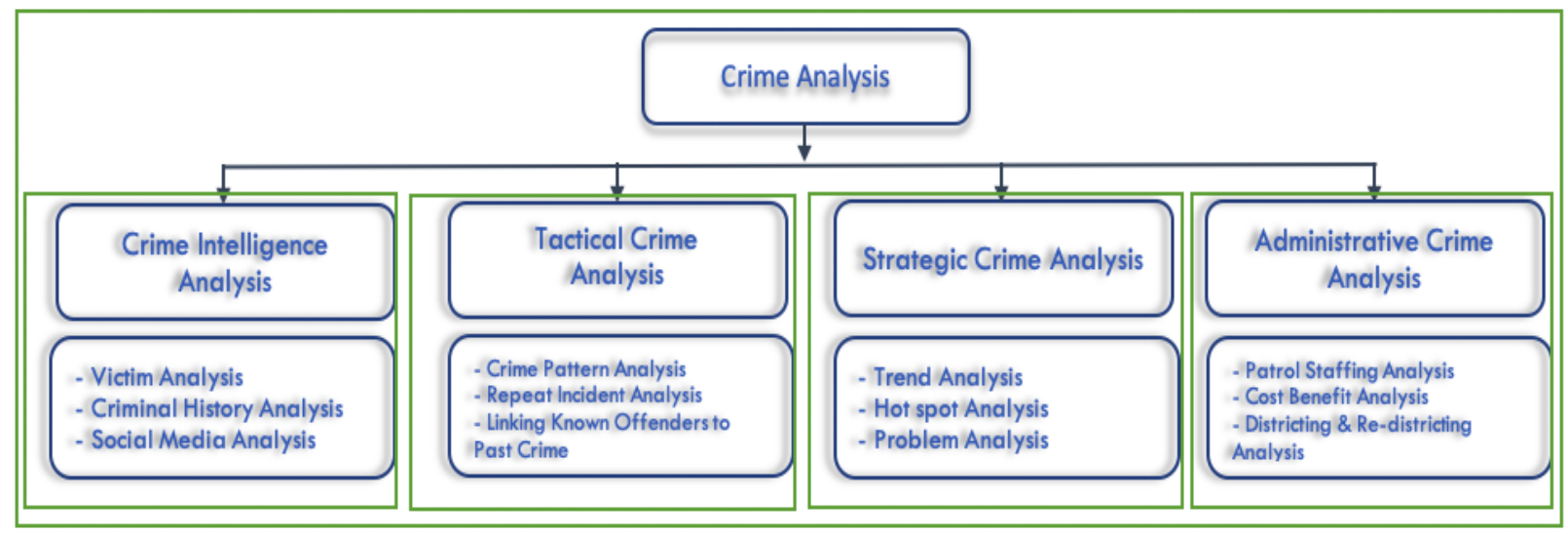

Figure 2 Types of Crime Analysis

The inception and development of trends in internet devices and facilities like Internet of Things (IoT) and Artificial Intelligence (AI) has helped both criminals and law enforcement agencies in various ways. As a consequence, law enforcement agencies have to double their effort through best optimization of technology to analyze, predict, solve and counter attack criminal activities by adopting new or emerging technologies. Hence, the application of different data analytics technologies to security and criminal investigation has immensely contributed to the development of criminal analytics [2]. Despite, the challenging issues of big data.

Relatively many scholars contributed to crime analysis and intelligent systems. From a statistical point of view, many used Principal Components Analysis (PCA) as a data analytic tool [11], [24], [25] to decrypt patterns of association between crimes, association or repetition of crime is a sign of multicollinearity of variables (i.e. represent the same Information), linear combinations of all variables are taken to reduce the number of variables from $\mathrm{p}$ to $\mathrm{m}$ to gain the information about an entire dataset via correlation between principle components and original variables. Fewaya et al, use PCA to examined the distribution of various crime and the overall criminality, degree of relationship between the distinct crimes and determined the distributary percentage of each crime in two Nigerian States [12].

Bhatal \& Dhiman compared three popular products in the big data domain; Cloudera CDH, Hortonworks HDP, and MapR highlighting their similarities, and distinct functionalities and present a visual representation of big data solution providers in the bid data analytic domain with Hadoop having the highest percentage [26];

Jain and Bhatnagar proposed a framework using Hadoop components to analyze and classify crime data based on many criteria such as total number of crimes from a range of years, total number of crime in each state, total number of crimes by type, and age and gender of the victims [7]. Pinto uses combiner to reduce the processing time in trend analysis [19].

Kirby and Macpherson proposed a similar framework in the journal titled integrating the National Intelligence Model with a problem-solving approach. The framework provides a technique that help in analyzing, developing and coordinating mechanism via multi agency collaboration that can deliver more sustainable and lasting solutions [27].

\section{METHODOLOGY}

In this work, various systems are analyzed and studied to have an understanding and proposed a new approach to crime analysis and intelligence model that will serve a large number of agencies making use of big data techniques. Data and information are collected through distinct method as various agencies are involved. However, due to the confidential nature of some information related to security and the fear of compromising that information some data are not revealed in the interviews that were granted. Common fact-finding techniques were used; interview, questionnaire, document analysis and review methodology for the literature review.

A. Interview was conducted to evaluate and investigate the existing method of crime data analysis with emphasis given to how data are used or treated in the analysis process. Interviews where accepted and granted across several divisions and department of the law enforcement agencies. Specifically, the divisional headquarter of the Nigeria Police Force Kano.

B. Document analysis involved reviewing various crime related document such as crime report, crime statistics, crime bulletin, security report, intelligence report and other important documents. The document analysis will provide tips for designing a correct schema or model of the system and will highlights the most important and prioritize piece of data that can be coupled with other sources to give an insight. It also helps in identifying the important attributes of all the individual objects in the class diagram.

C. Review methodology involves three important stages as the must easiest means of getting data. Crime related data and statistics is barely difficult to access due to the classification level of the data and the agencies involved. Therefore, the first stage involves the research of relevant works to crime data analysis especially in Nigerian context. It can be deduced that 
most of the literature reviewed in chapter 2 use various methodologies to analyzed crime data; however most of this method needs some improvement while others are not relevant due to the advent of new trend in crime analysis.

The review methodology reviewed various secondary and primary data sources ranging from journal articles, conference proceedings, big data and crime analysis reference books, big data tools and techniques documentation, white papers, interviews, excerpt, official websites are reviewed and cited where relevant.

\section{Data Sources}

There are number of agencies that deal with crime investigation and data. Each has its own method and jurisdiction of crime analysis or enforcement. Data gathered from these agencies can be treated as big data in the intelligence system model design. The most important and directly linked agencies are but not limited to the following;

\section{Force Criminal Investigation and Intelligence Department (FCIID)}

The FCIID is the highest investigation arm of the Nigerian Police force, its functions include investigation and prosecution of serious and complex criminal cases within and outside the country. it coordinates crime investigations/prosecution throughout the force. It involves various section or unit. Section of interest to this research include Central Criminal Registry (CCR), Special AntiRobbery Squad (SARS), X-Squad, General Investigation, Forensic Science, Interpol, Homicide and CIB \& SIB [28].

\section{Criminal Investigation Department (CID)}

The CID is a subset of the Nigeria Police Force that handles crime related cases hence, generating log of data daily. Other intelligent counterparts include Department of State Security (DSS), Nigeria Intelligent Agency (NIA).

\section{Federal Road Safety Commission}

The FRSC serve as a source of data through issuing the driver's license and keeping record of other road related offences of all citizens.

\section{Nigerian Prison Service}

The NPS has various data that can be integrated and used by other agencies especially the police and judiciary to track exconvicts record and determine total number of offences or crime committed by individual offenders.

\section{Nigerian Custom Service}

The Nigerian custom service has two important unit that generates most of its data namely; Custom Intelligent Unit (CIU) saddled with the responsibility of intelligent reports gathering. and ASYCUDA/Computer Specifically, the Unit handles Customs automation system in import clearance procedures called ASYCUDA, (Automated System for Customs Data), Its major features of interest include modules for Statistics, Documentation and Utilities [29].

\section{Nigerian Immigration Service}

The NIS also generate big data through managing and issuance of e-passport, visas and residence permit for foreigners and boarder surveillance and patrol [30]. The structured data can be used and shared in the system model design.

\section{National identity Management Commission}

NIMC served as a dedicated national database whose role is to enroll all Nigerians and foreigners with legal residents' permit. The commission is task with the following responsibilities [31];
1. Assign unique National Identification Number.

2. Incorporate identification number with other existing identity related database or registers.

3. Maintain secured communication with end users in public, private, organization, agencies and or body including card acceptance devices, or government centers.

4. Ensure preservation, protection, sanctity, and security of data collected (Cyber Security).

5. Respond to verification enquiries regarding identification of individuals.

6. Births and deaths registration.

\section{Other Data Sources}

Other data sources can be private organizations such as telecommunication companies, banks and other private companies that handle data. An API will be provided to enable easy access and integration.

\section{ANALYSIS MODEL AND DESIGN}

In this part the system model is fully described using various UML diagrams such as use case diagram, class diagram, deployment diagram and the designed system model prototype.

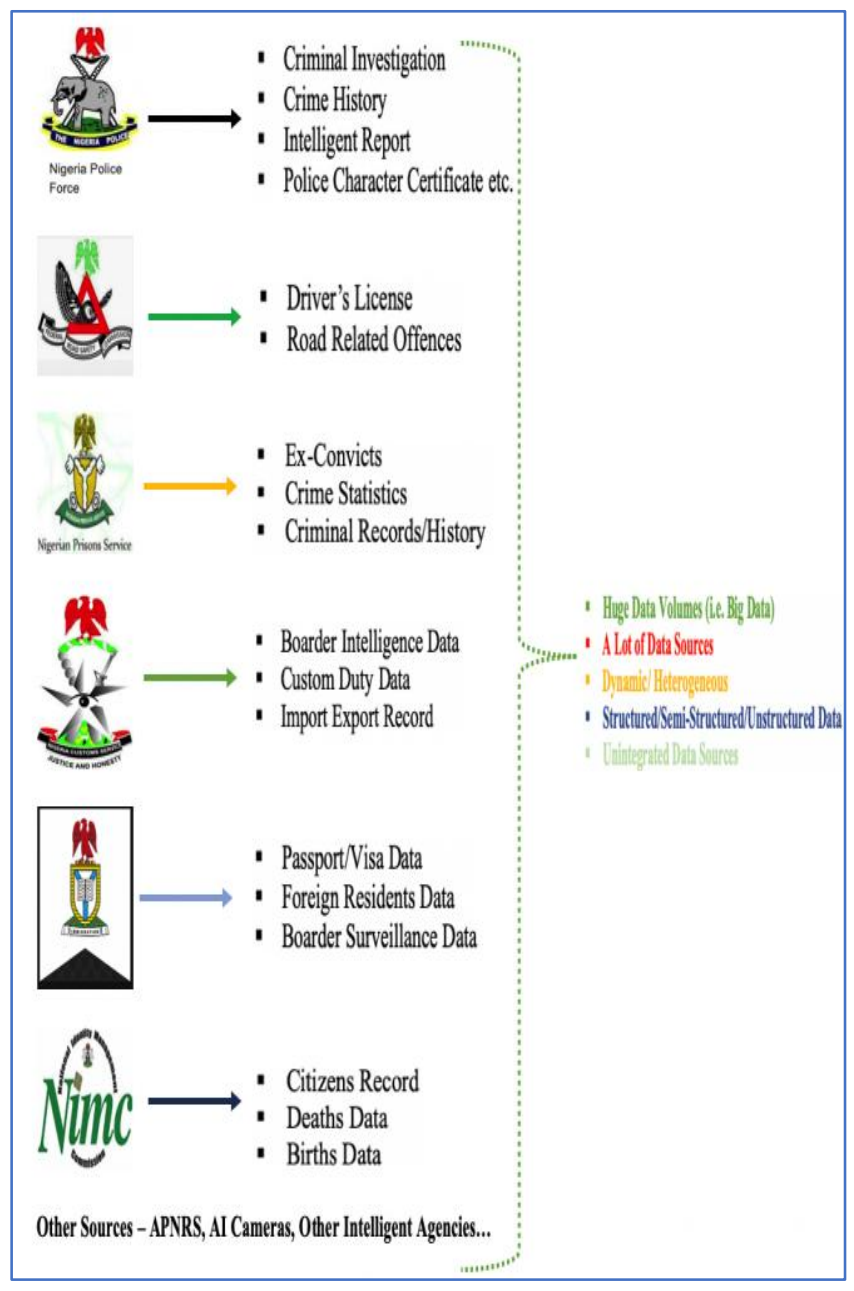

Figure 3 The System Model as Big Data

\subsection{Big Data and Model Description}

The figure 3 above summarizes how the model is treated or considered as big data and how the data possess the characteristics of big data such as volume, variety, velocity 
and other characteristics of big data. Data is generated at a very large scale with various agencies having sub-divisions or unit in over 36 States of the Federation each having a National and State headquarters with various mode of operations such as manual, file-based, automated and semi-automated systems.

$N P F$ is the sole agency for maintaining daily crimes in the society. NPF records and manage crime in various form especially crime data which include personal, job history and previous crime records if available. Daily statements from complainers are lodged to a large book log describing the nature of the event or crime in details. Police also keep record of crime scene and description such as the tools used in perpetrating the crime and the persons involved. Other crime records include finger print data of criminals which is also saved in a book log using ink and paper to thumb print the fingers. The agency also manages and issue police character certificate on request of individuals or other sister agencies.

FRSC is also a data source that holds a large number of national databases managing National Driver's License and road related offenses. The agency records basic citizen details liaison with other agencies like Vehicle Inspection Officers and health officers or hospitals. it holds biometric finger print data of millions of citizens and vehicle registration data. NPS the agency holds a large-scale data of imprisoned citizens and ex-convicts with their crime details and history of ex-convicts that were freed from the facility.

NCS this agency holds large database of imported items to the country and issue duty to owners of imported items. The agency holds database of boarder related information and intelligence report. Many units share and manage data at various points of entry in the country such as National sea ports, land boarders and airports nationwide. Intelligence unit of the service analyses and manage intelligence data of smuggled or illegal activities over the boarders and sea ports.
Hence, data is relatively generated and saved at an exponential rate.

NIS manages and issue national e-passport, visa and resident permit to foreigners or local residents. NIS holds a large database of citizens holding e-passport, diplomatic passport and visa. the Agency is also responsible for surveillance of national boarder and managing illegal immigrants and resident data.

NIMC sole commission tasked with the responsibility of capturing Nigerian citizens data and issuing a unique National Identification number. The agency is required to register in the national database over two hundred million record of Nigerians citizens. This include birth and death registration in various states of the federation and center of the agency. This database can further be treated as referenced point for other agencies of the federation such as Independent National Electoral Commission (INEC), Joint Admission and Matriculation Boards (JAMB), West African Examination Council (WAEC), Federal Inland Revenue Services (FIRS), National Drug and Law Enforcement Agency (NDLEA), National Health Insurance Scheme (NHIS), Federal Civil Service Commission, National Youth Service Corp (NYSC) and all other agencies that manage independent basic data of citizens. With the above integration data redundancy can be mitigated and National security can be enhanced through sim card registration and one-person-one-sim policy.

\subsection{Use Case Diagram}

A use case diagram model is used to describe and identify actors and functionality of the entire system model. Since various data sources and actors are involved as depicted in the methodology section of this research.

\subsection{Use Case Description}

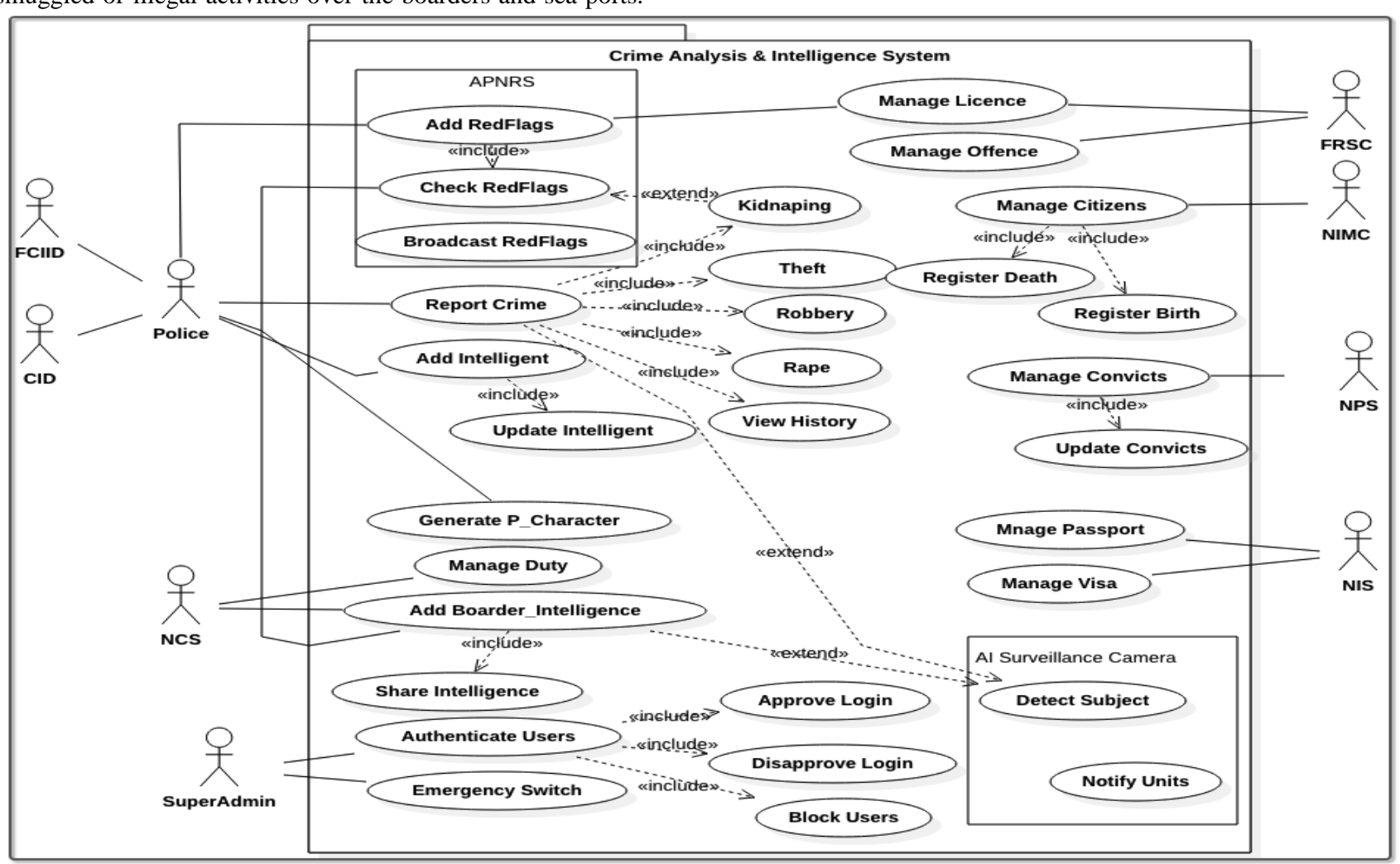

Figure 4 Use Case Diagram 


\section{Regular Users}

The figure above shows the complete use case diagram of the crime analysis and intelligence system model design. Considering the scope and boundaries of the system use case will have various and distinct actors as shown below;

- Nigeria Police Force

○ Criminal Investigation department (CID)

$\circ$ Force Crime Investigation and Intelligence Department (FCIID)

- Federal Road Safety Commission (FRSC)

- $\quad$ Nigeria Custom Service (NCS)

- $\quad$ Nigeria Prison Service (NPS)

- Nigeria Immigration Service (NIS)

- National Identity Management Commission (NIMC)

All the above will have the privilege of performing distinct functionalities and manage a cluster/server of data related to their state. Hence, total to thirty-six (36) administrators each accounting for single agency.

\section{SuperAdmin}

A single administrator responsible for managing all regular users considering the volatile and confidential nature of the system, various cyber security measures are implemented. On login, a hardware token is required by all regular users and approval by the superAdmin before a session of 1- or 2minutes elapse and random secret questions that can be only answered by fully authorized users. Error or failed login attempt is also considered a security breach attempt therefore warrant an automatic and immediate blockage of the account. Emergency switch is a preventive measure designed to partially switched off a cluster of the system in case of a major breach in the system security.

\section{System Actors and Use Cases}

There are two main system actors namely; Automatic Plate Number Recognition System (APNRS) and Artificial Intelligence Camera(s). They are important entity of the system that automatically detect and feed or notify relevant authorities of suspicious or red flags. Registered plate numbers on-contact while the AI embedded surveillance camera is fixed at designated strategic locations such as public places, main roads, religious building etc. to detect kidnapped individuals, wanted or suspicious individuals.

\subsection{Class Diagram}

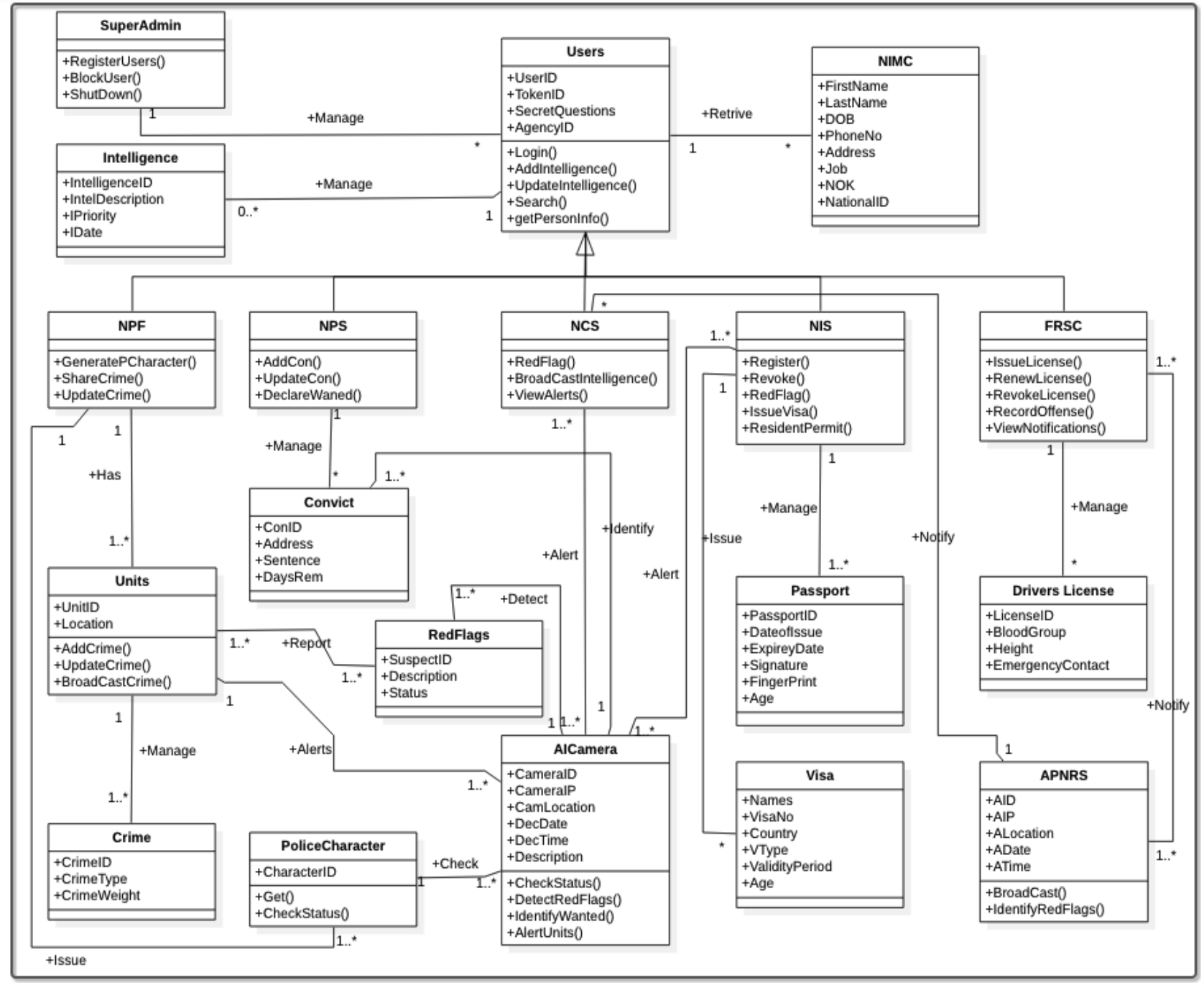

Figure 5 Class Diagram

The figure 5 above depicts the UML class diagram of the system. It includes all classes, attributes and their operations. 
The diagram shows the generalization relation of classes and association relationships with multiplicity or number of occurrences.

\subsection{Flowchart Diagrams}

A flowchart diagram is a diagrammatic representation that shows a workflow or represents a process. Regular user login is an important use case that requires a lot of process to ensure unauthorized access or system breach is prevented. The login process involved two actors (i.e. SuperAdmin and regularUser) as depicted in the flowchart below;

Step 1: A user initiates a login request and the system display a window requesting the user to generate a hardware token.

Step 2: If token is valid request approval is process by superAdmin, else the process is end. On approval the user is logged-in to the system successfully.

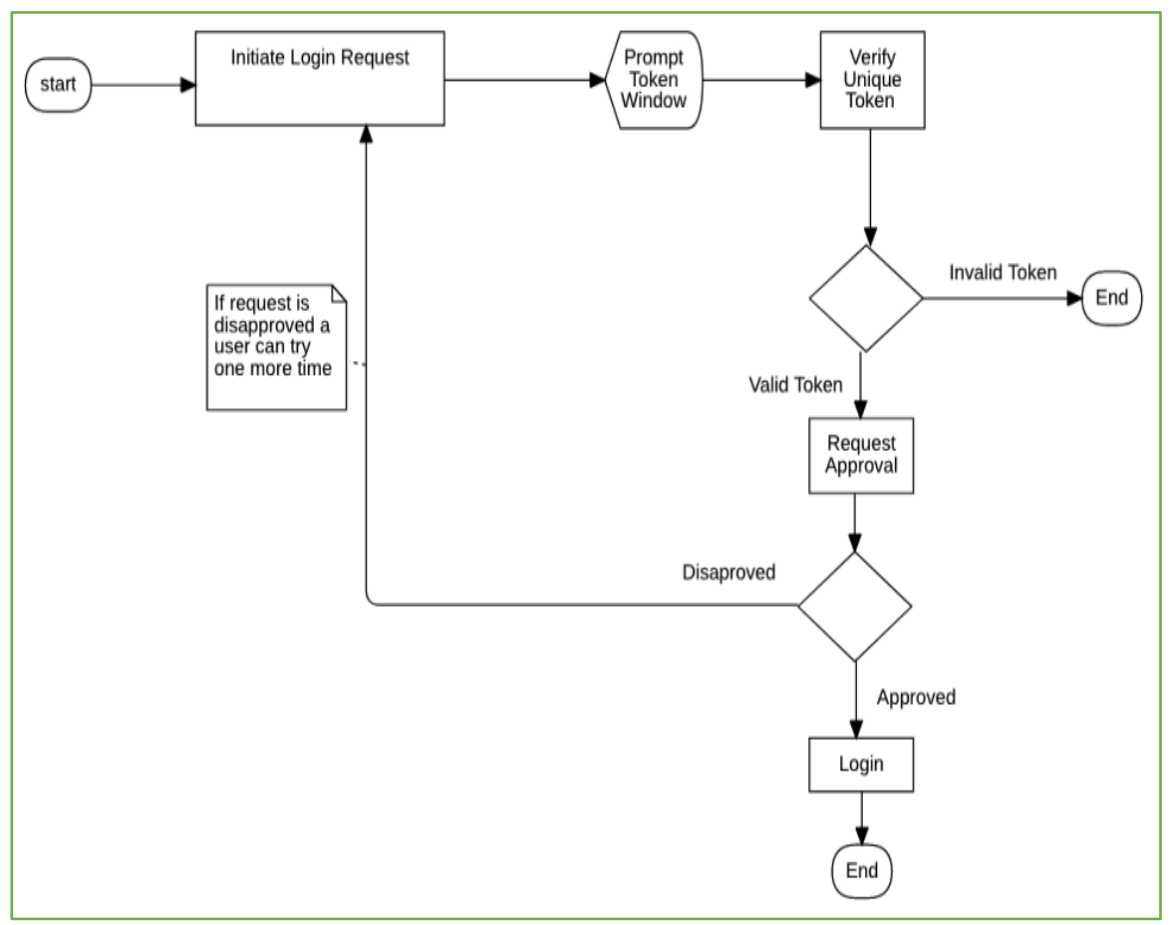

Figure 6 Log-In Flowchart

Add intelligence is a use case that allow all regular users to add intelligence to the system. The flow of the event is described in the steps below [figure 7]:

- Step 1: starts from a successful login to CAIS system using basic login procedure.

- Step 2: User click the "Add Intelligence" function and enter intelligence description, specify access level of the intelligence such as the agencies or other system actors that can access intelligence and then broadcast the intelligence to all specified agencies and system actors such as AI Camera and APNRS.

- Step 3: System Alert User Intelligence Added Successfully and current status of the intelligence.

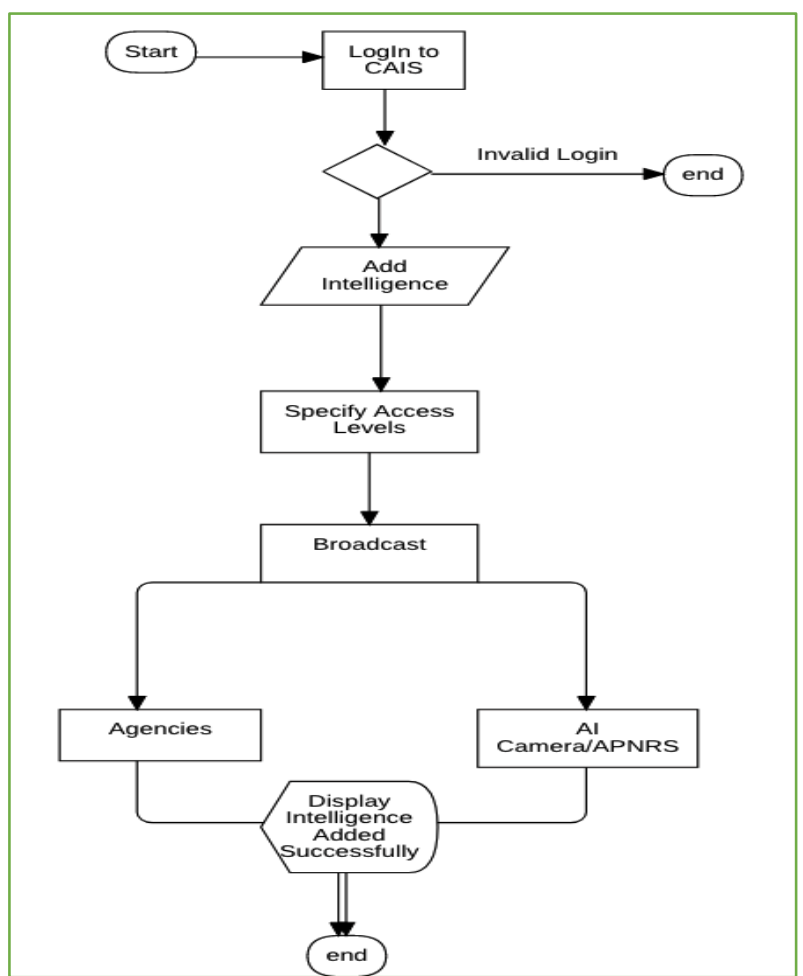

Figure 7 Add Intelligence Flowchart 
The figure 8 below shows a step-by-step process of adding a crime to CAIS. The process also starts by successful login by the user otherwise the process is terminated at this node or juncture.

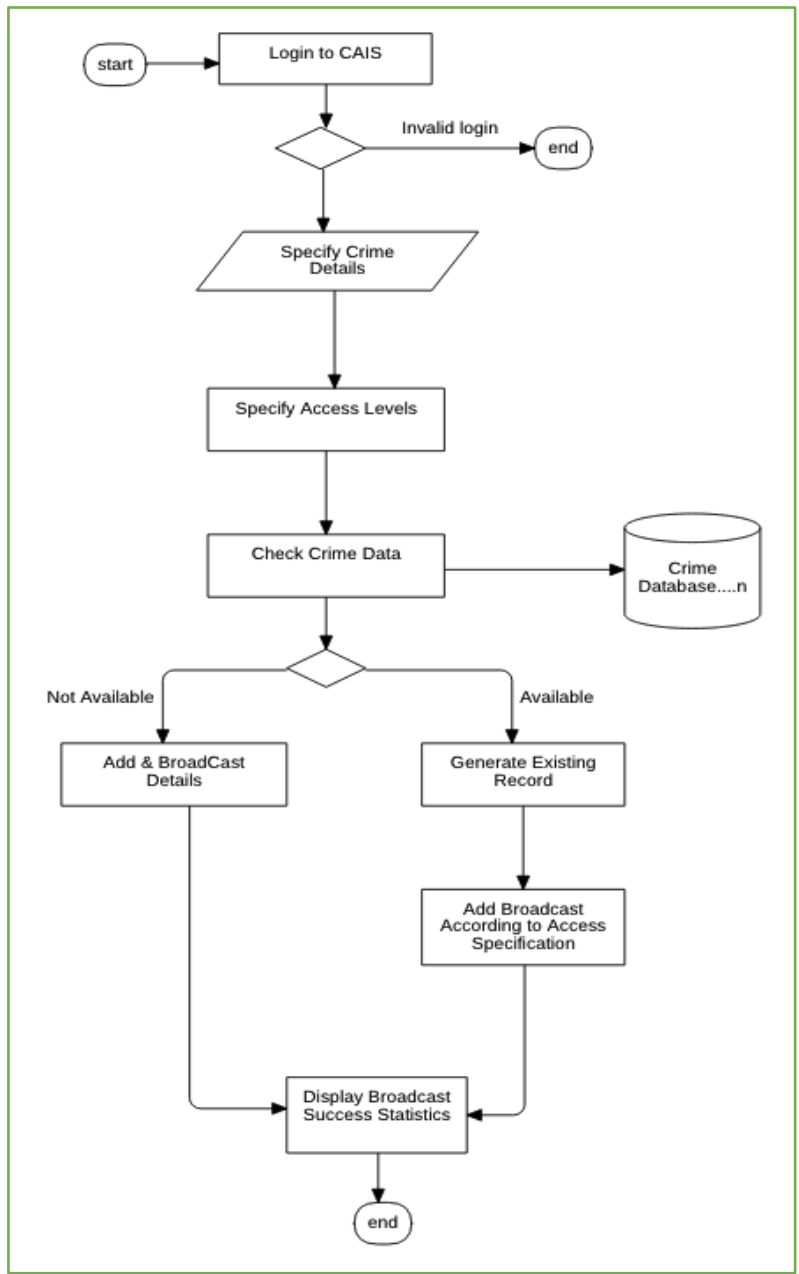

Figure 8 Add Crime Flowchart Diagram

- Step 1: User specify Crime details such as description, weight, contact details of the unit or officer handling the crime.

- Step 2: Specify access levels of the crime (i.e. restrict the agencies, users and systems) that can access the crime details.

- Step 3: Check crime data in other database of the system to confirm or retrieve other existing information about the new crime.

- Step 4a: if crime related data is available, retrieve details and add new details to CAIS.

- Step $4 b$ : if crime related data is not available, add new details to CAIS.

- Step 5: Display crime broadcast Statistics such as acknowledgment received by agencies.
The flowchart described the search redflags process in the steps in figure 9 ;

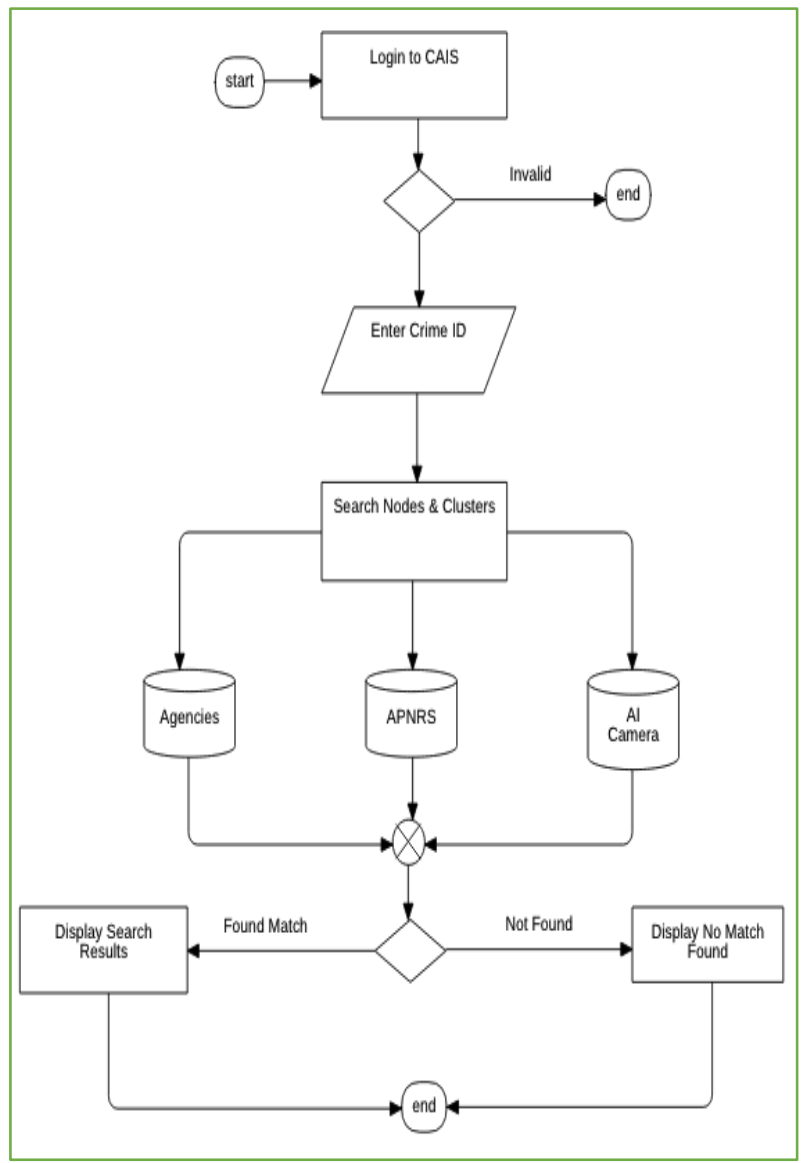

Figure 9 Search or Identify RedFlags Flowchart

- Step 1: Login to CAIS using valid credentials else terminate process.

- Step 2: Enter crime ID or Search phrase and select specifications to sort data and Select Nodes or databases to search e.g APNRS, AICamera, and CAIS.

- Step 3: Search nodes and clusters in the specified databases and merge and sort results accordingly.

- Step 4a: if found, Display Search Results.

- Step 4b: Not Found, display no existing record found.

\subsection{Deployment Diagram}

A deployment diagram is a graphical representation used to visualize the hardware, processors or nodes and devices of the model and the links of communication between the hardware and software files of the system. A node represents a physical entity that executes one or more components, subsystems or executables. A communication association is a straight line that shows the path of communication between nodes. The figure below shows the deployment diagram of Crime Analysis and Intelligence system model: 


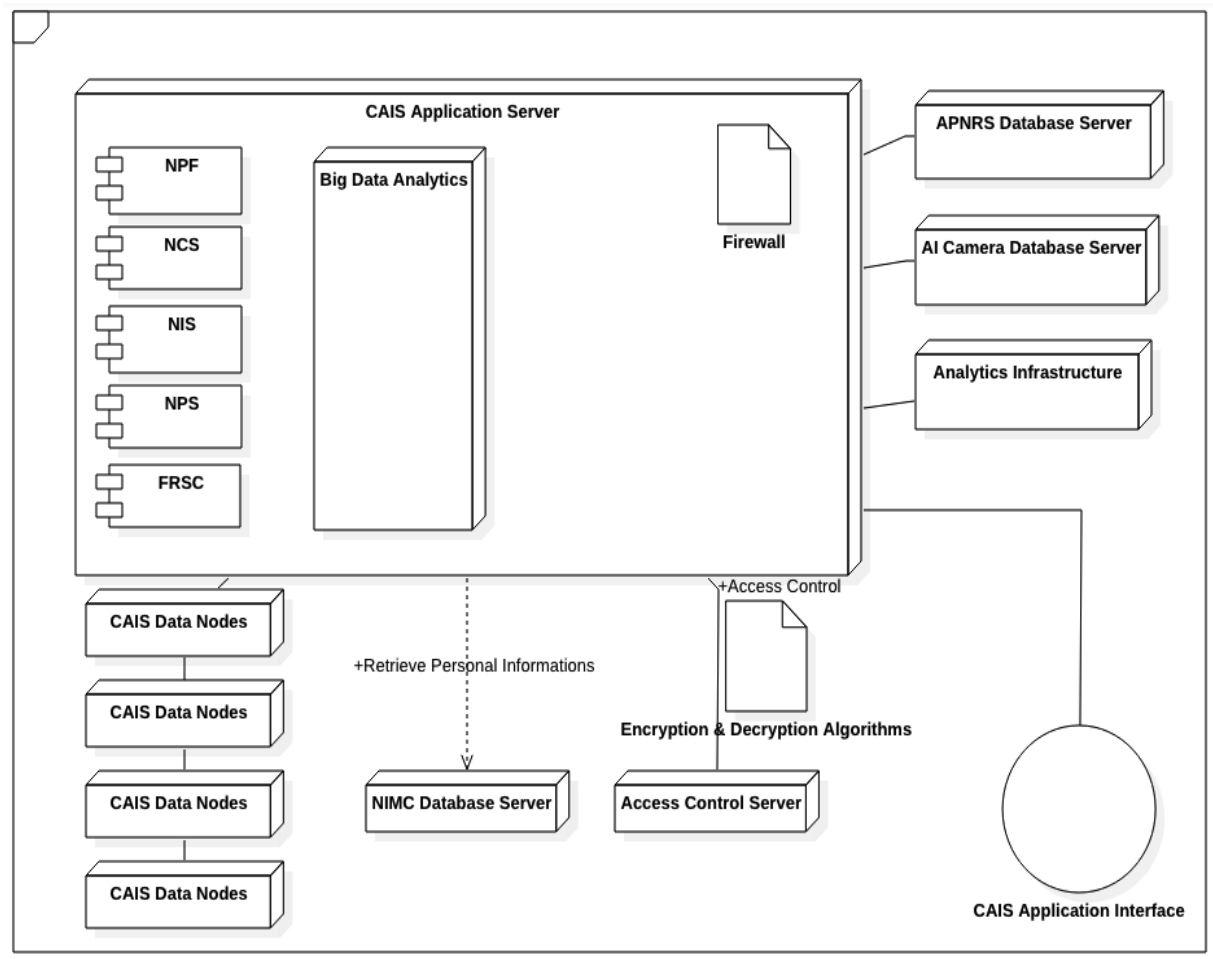

Figure 10 CAIS Deployment Diagram

CAIS application server as an integrated system has various modules or components that interactively add or share intelligence. The system has four major servers and many database nodes or clusters. The main server is the CAIS Application server which contains the agencies components. The main application server provides an API to all agencies to deploy their system modules. Information added to the system is uniquely tagged with an id representing the agency or user that added the information. A dependency relationship exists between the application server and NIMC Database server.

NIMC database server contain all citizen personal information and unique National Identification Number which is required by all participating agencies to register, search or identify citizens, achieved via an API provided by NIMC to CAIS. Other important node in CAIS application server is Big Data Analytics. Big Data Analytics and Analytics Infrastructure are the building block of the application that process data through the use of ETL (i.e. Extract Transform and Load). These infrastructures retrieve or extract data from various data source such as APNRS, AI Camera database; the data is then transformed into a designated data structure and finally loaded to CAIS data nodes.

Realtime data generated from APNRS and AI Camera database servers are feed to the analytics machine for sorting and merging. The data is then saved in CAIS data nodes. Hence, on retrieval or search in the application interface it can be visualized as a single piece of information generated from various source. Access control servers have a unidirectional relationship with the main server. it provides a secured control access to the main server through deploying firewalls and encryption \& decryption algorithms.

\section{CONCLUSION}

Firstly, in this study an in-depth analysis of the AS-IS system or processes was conducted. The results showed that most of agencies operate using manual filing system which involves transferring files from one office to the other and writing and distributing letters to police outpost and related agencies.
Personnel are also used to gather intelligent report and take all necessary actions.

The research also explored various big data techniques and analytic tools that can be readily outsourced and used for the purpose of crime analysis and intelligence sharing. The research further explore how various components of the Hadoop ecosystem is used to integrate and handle the large amount of data generated at various level, and how this data can be broadcasted or shared among sister agencies timely and efficiently, as time is of utmost importance in crime analysis and intelligence sharing.

The proposed model designed is represented using the most important unified modelling languages tools: use case diagram to identify the use cases of the system and describe the behavior of the system, class diagram to represent the attributes, operations, relationships between classes and the number or multiplicity of occurrences, flowchart to show the flow of a use case, deployment diagram to depict both hardware and software components of the model and how they are related.

In Future works: a prototype design and implementation of the system model will be designed to show the final output of the research. Denoting the various features of the system such as add intelligence, analyzed crime, view redflags, and many more. Subsequently,

1. A case study of the Nigerian security agencies will be researched and real-time data will be sourced and analyzed to check the effectiveness of the proposed model design.

2. Test the use of other hardware components of the model such as APNRS, and AI Camera using realtime data.

\section{REFERENCES}

[1] V. Dhar, 'Data Science and Prediction', Commun. acm, vol. 56, no. 12, 2013.

[2] M. I. Pramanik, R. Y. K. Lau, W. T. Yue, Y. Ye, and C. 
$\mathrm{Li}$, 'Big data analytics for security and criminal investigations', Wiley Interdiscip. Rev. Data Min. Knowl. Discov., vol. 7, no. 4, pp. 1-19, 2017.

[3] D. Tyagi and S. Sharma, 'an Approach To Crime Data Analysis: a Systematic Review', Int. J. Eng. Technol. Manag. Res., vol. 5, no. 2, pp. 67-74, 2018.

[4] A. Barbutu, 'Big Data and Policing An Assessment of Law Enforcement Requirements, Expectations and Priorities', 2017.

[5] N. Aarathi Srinivas, N. Gayathri, R. Indraja, S. Srividhya, and J. Kayalvizhi, 'Crime Analysis and Prediction Uisng Big Data', Int. J. Pure Appl. Math., vol. 119, no. 12, pp. 207-211, 2018.

[6] R. Sanjana, H. S. S, and S. Sruthi, 'Big Data Approach for Crime Classification and Visualization Using Crime Dataset', Int. J. Innov. Res. Sci. Eng. Technol., vol. 7, no. 2, pp. 25-29, 2018.

[7] A. Jain and V. Bhatnagar, 'Crime Data Analysis Using Pig with Hadoop', Procedia Comput. Sci., vol. 78, no. December 2015, pp. 571-578, 2016.

[8] N. G. Karthigaa and M. K. B. Janani, 'Big Data Prediction on Crime Detection', in National Conference on Computational Intelligence Systems, 2017, pp. 43-48.

[9] V. N. Inukollu, S. Arsi, and S. Rao Ravuri, 'Security Issues Associated with Big Data in Cloud Computing', Int. J. Netw. Secur. Its Appl., vol. 6, no. 3, pp. 45-56, 2014.

[10] H. V. Jagadish et al., 'Big data and its technical challenges', Commun. ACM, vol. 57, no. 7, pp. 86-94, 2014.

[11] D. C. Ukpabi, 'Statistical Analysis on Crime Rate in Nigeria', 2018.

[12] O. Faweya, A. T. Adeniran, and K. O. Balogun, 'Principal Component Analysis of Crime Rate in Nigeria : A Case Study of Ekiti and Osun State', Am. J. Math. Stat., vol. 8, no. 4, pp. 79-88, 2018.

[13] Sheriff Deputies, 'The Anatomy of Crimes in Nigerial: A Statistical Study', 2015. [Online]. Available: http://www.sheriffdeputiesltd.com/crimes-in-nigeria/. [Accessed: 09-Jan-2020]

[14] A. Hills, 'Partnership policing: Is it relevant in Kano, Nigeria?', Criminol. Crim. Justice, vol. 14, no. 1, pp. 824, Feb. 2014.

[15] S. Rewari and W. Singh, 'S ystematic R eview of C rime $\mathrm{D}$ ata A nalytics', in IEEE International Conference on Power, Control, Signals and Instrumentation Engineering (ICPCSI-2017), 2017, pp. 3042-3045.

[16] Nurhayati, Busman, and V. Amrizal, 'Big Data Analysis Using Hadoop Framework and Machine Learning as Decision Support System (DSS) (Case Study: Knowledge of Islam Mindset)', in 2018 6th International Conference on Cyber and IT Service Management, CITSM 2018, 2018, no. Citsm, pp. 1-6.

[17] J. C. Pennsylvania, 'Big Data and NoSQL', 2019. [Online]. Available: http://jcsites.juniata.edu/faculty/rhodes/dbms/nosql.htm. [Accessed: 09-Jan-2020].
[18] M. P.K, 'Technology's Ways: Information Technology, crime analysis and the rationalizing of policing', SAGE, vol. 1, no. 1, pp. 83-103, 2015.

[19] K. Overland Park, 'Definition and Types of Crime Analysis (White Paper 2014-02)', 2014.

[20] V. F. Pinto, 'Trend Analysis using Hadoop' s MapReduce Framework', in 2017 2nd International Conference on Computational Systems and Information Technology for Sustainable Solution (CSITSS), 2017, pp. $1-5$.

[21] M. Feng, J. Zheng, Y. Han, J. Ren, and Q. Liu, 'Big Data Analytics and Mining for Crime Data Analysis, Visualization and Prediction', in Big Data Analytics and Mining for Crime Data Analysis, Visualization and Prediction: 9th International Conference, BICS 2018, Xi'an, China, July 7-8, 2018, Proceedings, 2018, vol. 10989 LNAI, no. July, pp. 605-614.

[22] A. Oguntimilehin and O. Ademola, 'A Review of Big Data Management, Benefits and Challenges A Review of Big Data Management, Benefits and Challenges', $J$. Emerg. Trends Comput. Inf. Sci., vol. 5, no. July 2014, pp. 433-438, 2015.

[23] P. Merla, 'Data Analysis using Hadoop MapReduce Environment', in IEEE International Conference on Big Data (BIGDATA), 2017, pp. 4783-4785.

[24] J. Joseph and J. Corkill, 'Information evaluation: how one group of intelligence analysts go about the task', in Australian Security and Intelligence Conference Conferences, 2011, pp. 97-103.

[25] O. O. O, Akintande O J, and Ekum M, 'Analyzing the Distribution of Crimes in Oyo State (Nigeria) using Principal Component Analysis (PCA)', IOSR J. Math. Ver. I, vol. 11, no. 3, pp. 2278-5728, 2015.

[26] P. E. Oguntunde, O. O. Ojo, H. I. Okagbue, and O. A. Oguntunde, 'Analysis of selected crime data in Nigeria', Data Br., vol. 19, pp. 1242-1249, 2018.

[27] G. Bhathal and A. S. Dhiman, 'Big Data Solution: Improvised Distributions Framework of Hadoop', in 2018 Second International Conference on Intelligent Computing and Control Systems (ICICCS), 2018, no. Iciccs, pp. 35-38.

[28] I. Mcpherson and S. Kirby, 'intelligence and problem solving Integrating the National Intelligence Model with a " problem solving " approach', Community Saf. J., vol. 3, no. 2, pp. 36-46, 2004.

[29] T. N. P. Force, 'Nigeria Police Force', 2020. [Online]. Available: https://www.npf.gov.ng.

[30] NCS, 'Special commands units', Nigerian Custom Service. [Online]. Available: https://customs.gov.ng/?page_id=3113. [Accessed: 11Mar-2020].

[31] NIS, 'Our Core Mandate', NIS, 2020. [Online]. Available: https://immigration.gov.ng/nis-core-mandate/. [Accessed: 11-Mar-2020].

[32] NIMC, 'Functions/Powers of NIMC', NIMC, 2019. [Online]. Available: https://www.nimc.gov.ng/functionspowers/. [Accessed: 12-Mar-2020]. 